\title{
High-order difference potentials methods for 1D elliptic type models
}

\author{
Yekaterina Epshteyn and Spencer Phippen \\ Department of Mathematics, The University of Utah, Salt Lake City, UT, 84112
}

\begin{abstract}
Numerical approximations and modeling of many physical, biological, and biomedical problems often deal with equations with highly varying coefficients, heterogeneous models (described by different types of partial differential equations (PDEs) in different domains), and/or have to take into consideration the complex structure of the computational subdomains. The major challenge here is to design an efficient and flexible numerical method that can capture certain properties of analytical solutions in different domains/subdomains (such as positivity, different regularity/smoothness of the solutions, etc), while handling the arbitrary geometries and complex structures of the domains. In this work, we employ onedimensional elliptic type models as the starting point to develop and numerically test highorder accurate Difference Potentials Method (DPM) for variable coefficient elliptic problems in heterogeneous media. While the method and analysis are simple in the one-dimensional settings, they illustrate and test several important ideas and capabilities of the developed approach.
\end{abstract}

Keywords: Difference potentials, boundary projections, Cauchy's type integral, boundary value problems, variable coefficients, heterogeneous media, high-order finite difference schemes, Difference Potentials Method, Immersed Interface Method, interface problems, parallel algorithms

$M S C: 65 \mathrm{~N} 06,65 \mathrm{~N} 12,65 \mathrm{~N} 15,65 \mathrm{~N} 22,65 \mathrm{~N} 99$

\section{Introduction}

Numerical approximations and modeling of many physical, biological, and biomedical problems often deal with equations with highly varying coefficients, heterogeneous models, and/or have to take into consideration the complex structure of the computational subdomains. The major challenge here is to design an efficient and flexible numerical method (for example, multi-scale method) that can capture certain properties of analytical solutions in different domains/subdomains, while handling the arbitrary geometries and complex structures of the domains.

There is extensive literature that addresses problems in domains with irregular geometries 
and interface problems. Some established finite-difference based methods for such problems are the Immersed Boundary Method (IB) ([17, 18], etc), the Immersed Interface Method (IIM) ([10, 9, 11], etc), the Ghost Fluid Method (GFM) ([5], [12], [13], etc), the Matched Interface and Boundary Method (MIB) ([32, 30, 31], etc), and the method based on the Integral Equations approach, ([15], etc). These methods are robust sharp interface methods that have been applied to solve many problems in science and engineering. For a detailed review of the subject the reader can consult [11].

We consider here an approach based on Difference Potentials Method (DPM) [22, 24]. The DPM on its own, or in combination with other numerical methods, is an efficient tool for the numerical solution of interior and exterior boundary value problems in arbitrary domains (see for example, [22, 24, 14, 25, 28, 16, 23, 27, 3, 4]). Viktor S. Ryaben'kii originally introduced DPM in his Doctor of Science thesis (Habilitation thesis) in 1969. The DPM allows one to reduce uniquely solvable and well-posed boundary value problems to pseudo-differential boundary equations with projections. Similar to the method in [15], methods based on Difference Potentials (see for example [22], [23, 27, 4], [16], etc) introduce computationally simple auxiliary domains. After that, the original domains/subdomains are embedded into simple auxiliary domains (and the auxiliary domains are discretized using Cartesian grids). However, compared to the integral equation approach in [15], methods based on Difference Potentials construct discrete pseudo-differential Boundary Equations with Projections to obtain the values of the solutions at the points near the continuous boundaries of the original domains (at the points of the discrete grid boundaries which approximate the continuous boundaries from the inside and outside of the domains). Using the obtained values of the solutions at the discrete grid boundaries, the approximation to the solution in each domain/subdomain is constructed through the discrete generalized Green's formulas.

The main complexity of the methods based on Difference Potentials approach reduces to several solutions of simple auxiliary problems on structured Cartesian grids. Like the method in [15], and IIM, GFM and MIB, methods based on Difference Potentials preserve the underlying accuracy of the schemes being used for the space discretization of the continuous PDEs in each domain/subdomain. But compared to [15], and to IIM and GFM, methods based on Difference Potentials are not restricted by the type of the boundary or interface conditions (as long as the continuous problems are well-posed), see [22] or some example of the recent works [2], [23, 27, 4], ect. Furthermore, DPM is computationally efficient since any change of the boundary/interface conditions affects only a particular component of the overall algorithm, and does not affect most of the numerical algorithm (this property of the numerical method is crucial for computational and mathematical modeling of many applied problems). Finally, Difference Potentials approach is well-suited for the development of parallel algorithms, see [23, 27, 4] - examples of the second-order in space schemes based on Difference Potentials idea for $2 D$ interface/composite domain problems. The reader can consult $[22,24]$ and $[19,20]$ for a detailed theoretical study of the methods based on Difference Potentials, and $([22,24,14,25,28,26,16,2,23,27,3,4]$, etc) for the recent developments and applications of DPM.

In this work, we employ one-dimensional elliptic type models (second-order Boundary 
Value Problem (BVP)) as the starting point, to develop and numerically test high-order accurate methods based on Difference Potentials approach for variable coefficient elliptic type problems in heterogeneous media. Let us note that, previously in [23, 27, 4], we have developed efficient (second-order accurate in space) schemes based on Difference Potentials idea for $2 D$ interface/composite domain problems. The method developed in $[23,27,4]$ can handle non-matching interface conditions (as well as non-matching grids between each subdomain), and is well-suited for the design of parallel algorithms. However, these schemes were constructed and tested for the solution of the Poisson's or heat equations (constant coefficient). Also, a different example of the efficient and high-order accurate method, based on Difference Potentials for the Helmholtz equation in homogeneous media with the variable wave number, was recently developed and numerically tested in [16] for a single 2D domain. But to the best of our knowledge, this is the first application (at this point in the simple settings) of Difference Potentials approach for the construction of high-order accurate numerical schemes for problems with variable coefficients in heterogeneous media and non-matching interface conditions. While the method and analysis are simple in the one-dimensional setting, they illustrate and test several important ideas and capabilities of Difference Potentials approach. Furthermore, to develop these methods, we employ here a more general viewpoint on Difference Potentials of being discrete potentials for the linear difference schemes (rather than approximation to the surface potentials $[19,20]$ ) - "Difference Potential plays the same role for the solution of a general system of linear difference equations (linear difference scheme), as the classical Cauchy's type integral for the solution of Cauchy-Riemann system, or in other words for the analytic functions" - see [24] or see Sections 4.1 and 8.

The paper is organized as follows. First, in Section 2 we give a brief summary of the main steps of the proposed algorithms. In Section 3 we introduce the formulation of our problem. Next, to illustrate the framework for the construction of DPM with a different order of accuracy, we construct DPM with a second and with a fourth-order accuracy in Section 4.1 for a single domain $1 D$ elliptic type model. In Section 5, we extend the second and the fourth-order DPM to one-dimensional elliptic type interface/composite domain model problem. Finally, we illustrate the performance of the proposed Difference Potentials Methods, as well as compare Difference Potentials Methods with the Immersed Interface Method in several numerical experiments in Section 6. Some concluding remarks are given in Section 7.

\section{Algorithm}

In this section we will briefly summarize the main steps of our algorithm. We will give a detailed description of each step in the subsequent sections below.

- Step 1: Introduce a computationally simple auxiliary domain and formulate the auxiliary problem (AP). 
- Step 2: Compute a Particular solution, $u_{j}:=G^{h} f, x_{j} \in N^{+}$, as the solution of the Auxiliary Problem (AP). For the single domain method, see (4.12) - (4.13) in Section 4.1 (second-order and fourth - order method). For the straightforward extension of the algorithms to the interface and composite domains problems, see Section 5.

- Step 3: Next, compute the unknown boundary values or densities $u_{\gamma}$ at the points of the discrete grid boundary $\gamma$ (value of the unknown density $u_{\gamma}$ on $\gamma$ ) by solving the system of linear equations derived from the system of Boundary Equations with Projection: see (4.31) - (4.32) (second - order method), or (4.35) - (4.36) (fourth - order method) in Section 4.1, and extension to the interface and composite domain problems (5.2) (5.3) in Section 5.

- Step 4: Using the definition of the difference potential, Def. 4.2, Section 4.1, and Section 5 (algorithm for interface/composite domain problems), construct the Difference Potential $\mathbf{P}_{N^{+}{ }_{\gamma}} u_{\gamma}$ from the obtained density $u_{\gamma}$.

- Step 5: Finally, reconstruct the approximation to the continuous solution from $u_{\gamma}$ using the generalized Green's formula $u(x) \approx \mathbf{P}_{N^{+} \gamma} u_{\gamma}+G^{h} f$, see Theorem 4.4 in Section 4.1, and see Theorem 5.1 in Section 5.

\section{Elliptic type interface models}

We are concerned here with a 1D elliptic type interface problem of the form:

$$
\begin{aligned}
& \left(k_{1} u_{x}\right)_{x}-\sigma_{1} u=f_{1}, \quad x \in I_{1}, \\
& \left(k_{2} u_{x}\right)_{x}-\sigma_{2} u=f_{2}, \quad x \in I_{2},
\end{aligned}
$$

subject to the Dirichlet boundary conditions specified at the points $x=0$ and $x=1$ :

$$
u(0)=a, \text { and } u(1)=b
$$

and interface conditions at $\alpha$ :

$$
l_{\text {int }}(u)=\phi, \quad x=\alpha
$$

where $I_{1}:=[0, \alpha) \subset I_{1}^{0}$ and $I_{2}:=(\alpha, 1] \subset I_{2}^{0}$ are two subdomains of the domain $I:=[0,1]$, $0<\alpha<1$ is the interface point, and $I_{1}^{0}$ and $I_{2}^{0}$ are some auxiliary subdomains that contain the original subdomains $I_{1}$ and $I_{2}$ respectively. The functions $k_{1}(x) \geq 1, k_{2}(x) \geq 1, \sigma_{1}(x) \geq$ $0, \sigma_{2}(x) \geq 0$ are sufficiently smooth functions defined in a larger auxiliary subdomains $I_{1}^{0}$ and $I_{2}^{0}$, respectively. $f_{1}(x)$ and $f_{2}(x)$ are sufficiently smooth functions defined in each subdomain $I_{1}$ and $I_{2}$ respectively. Note, we assume that the operator on the left-hand side of the equation (3.1) is well-defined on some larger auxiliary domain $\overline{I_{1}^{0}}$, and the operator on the left-hand side of the equation (3.2) is well-defined on some larger auxiliary domain $\overline{I_{2}^{0}}$. More precisely, we assume that for any sufficiently smooth functions on the right-hand side of (3.1 ) - (3.2), the equations (3.1) and (3.2) have a unique solution on $I_{1}^{0}$ and $I_{2}^{0}$, that satisfy the given boundary conditions on $\partial I_{1}^{0}$ and $\partial I_{2}^{0}$, respectively.

Remark: The Dirichlet boundary conditions (3.3) are chosen only for the purpose of illustration and the method (DPM) is not restricted by any type of boundary conditions. 


\section{Single domain}

Our goal is to develop high-order methods based on Difference Potentials idea for the problem (3.1) - (3.4). To simplify the presentation (and to illustrate the unified framework for the construction of DPM with different orders of accuracy for the problems in single domain, and/or for the interface/composite domain problems), we will first state the second and the fourth-order methods for the single domain problem:

$$
\left(k u_{x}\right)_{x}-\sigma u=f, \quad x \in I
$$

subject to the Dirichlet boundary conditions specified at the points $x=0$ and $x=1$ :

$$
u(0)=a, \text { and } u(1)=b,
$$

and then extend the developed ideas in a straightforward way to the interface/composite domain problem (3.1) - (3.4) in Section 5. As before, $I=[0,1]$, the functions $k(x) \geq 1$, $\sigma(x) \geq 0$ are sufficiently smooth functions defined in some auxiliary domain $I^{0}$, such that $I \subset I^{0}$ and $f(x)$ is sufficiently smooth function defined in $I$. We also assume that the model problem (4.1) - (4.2) is well-posed, as well as that the operator on the left-hand side of the equation (4.1) is well-defined on some larger auxiliary domain $\overline{I^{0}}$. Similar to [22, 23, 27, 24], let us now introduce and define the main steps of the DPM for this problem.

\subsection{Difference potentials approach for construction of high-order methods}

We will present below (at this point, using simple one-dimensional settings) a framework based on Difference Potentials approach to construct high-order methods for problems with variable coefficients in heterogeneous media, and non-matching interface conditions. However, major principles of this framework will stay the same when applied to the numerical approximation of the models in arbitrary domains in $2 \mathrm{D}$ and $3 \mathrm{D}$, and subject to general boundary conditions. Also, it is important to note that the presented approach based on Difference Potentials is general, and can be employed in similar ways with any (most suitable) underlying high-order discretization of the given continuous problem. In this work, the particular choices of the second-order discretization (4.6) and the fourth-order discretization (4.7) were only employed for purpose of the efficient illustration and implementation of the ideas.

We will present our ideas below by designing the second-order and the fourth-order methods together, and will only comment on the technical differences.

\section{Introduction of the Auxiliary Domain:}

Let us place the original domain $I$ in the auxiliary domain $I^{0}:=[c, d] \subset \mathbb{R}$. Next, we introduce a Cartesian mesh for $I^{0}$, with points $x_{j}=c+j \Delta x,\left(j=0,1, \ldots, N^{0}\right)$. Let us assume for simplicity that $\Delta x:=h=\frac{d-c}{N^{0}}$. Note that the boundary points $x=0$ and 


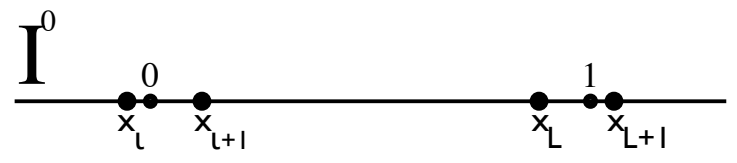

Figure 1: Example (a sketch) of the auxiliary domain $I^{0}$, original domain $I=[0,1]$, and the example of points in set $\gamma=\left\{x_{l}, x_{l+1}, x_{L}, x_{L+1}\right\}$ for the 3 -point second-order scheme.

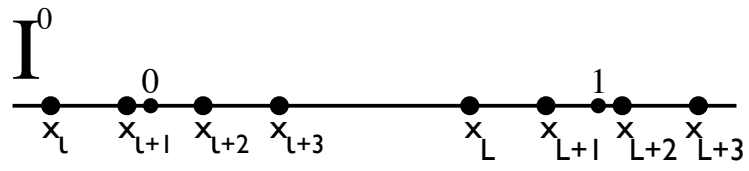

Figure 2: Example (a sketch) of the auxiliary domain $I^{0}$, original domain $I=[0,1]$, and the example of points in set $\gamma=\left\{x_{l}, x_{l+1}, x_{l+2}, x_{l+3}, x_{L}, x_{L+1}, x_{L+2}, x_{L+3}\right\}$ for the 5-point fourth-order scheme.

$x=1$ will typically fall between grid points, say $x_{l} \leq 0 \leq x_{l+1}$ and $x_{L} \leq 1 \leq x_{L+1}$ (for the 3-point second order scheme); and between grid points $x_{l}<x_{l+1} \leq 0 \leq x_{l+2}<x_{l+3}$ and $x_{L}<x_{L+1} \leq 1 \leq x_{L+2}<x_{L+3}$ (for the 5 -point fourth order scheme), see Figure 1 and Figure 2 .

Now, we define a finite-difference stencil $N_{j}^{\kappa}:=N_{j}^{3}$ or $N_{j}^{\kappa}:=N_{j}^{5}$ with its center placed at $x_{j}$, to be a 3-point central finite-difference stencil of the second-order method, or a 5-point central finite-difference stencil of the fourth-order method, respectively:

$$
\begin{gathered}
N_{j}^{\kappa}:=\{j-1, j, j+1\}, \quad \kappa=3, \text { or } \\
N_{j}^{\kappa}:=\{j-2, j-1, j, j+1, j+2\}, \quad \kappa=5
\end{gathered}
$$

Next, we introduce point set $M^{0}$, the set of all the grid nodes $x_{j}$ that belong to the interior of the auxiliary domain $I^{0} ; M^{+}:=M^{0} \cap I$, the set of all the grid nodes $x_{j}$ that belong to the interior of the original domain $I$; and $M^{-}:=M^{0} \backslash M^{+}$, the set of all the grid nodes $x_{j}$ 
that are inside of the auxiliary domain $I^{0}$, but belong to the exterior of the original domain I. Define $N^{+}:=\left\{\bigcup_{j} N_{j}^{\kappa} \mid x_{j} \in M^{+}\right\}$, the set of all points covered by the stencil $N_{j}^{\kappa}$ when the center point $x_{j}$ of the stencil goes through all the points of the set $M^{+} \subset I$. Similarly, define $N^{-}:=\left\{\bigcup_{j} N_{j}^{\kappa} \mid x_{j} \in M^{-}\right\}$, the set of all points covered by the stencil $N_{j}^{\kappa}$ when the center point $x_{j}$ of the stencil goes through all the points of the set $M^{-}$.

Now we can introduce the set $\gamma:=N^{+} \cap N^{-}$. The set $\gamma$ is called the discrete grid boundary. The mesh nodes from set $\gamma$ straddle the boundary $\partial I \equiv\{0,1\}$. In case of the second-order method (with 3 - point stencil), the set $\gamma$ will contain four mesh nodes $\gamma=\{l, l+1, L, L+1\}$, see Figure 1. In case of the fourth-order method (with 5 - point stencil), the set $\gamma$ will contain eight mesh nodes $\gamma=\{l, l+1, l+2, l+3, L, L+1, L+2, L+3\}$, see Figure 2. Finally, define $N^{0}:=\left\{\bigcup_{j} N_{j}^{\kappa} \mid x_{j} \in M^{0}\right\} \subset \overline{I^{0}}$.

Once again, let us emphasize, that $\kappa$ either takes here the value 3 (if the 3-point stencil is used to construct the second-order method), or 5 (if the 5-point stencil is used to construct the fourth-order method).

The point sets $N^{0}, M^{0}, N^{+}, N^{-}, M^{+}, M^{-}, \gamma$ will be used to develop high-order methods based on the Difference Potentials idea.

\section{Construction of Difference Equations:}

The discrete version of the problem (4.1) is to find $u_{j} \in N^{+}$such that

$$
L_{h}\left[u_{j}\right]=f_{j}, \quad x_{j} \in M^{+}
$$

The discrete system of equations (4.5) is obtained here by discretizing (4.1) with the standard second-order 3-point central finite difference scheme (4.6) (if the second-order accuracy is desired), or with the fourth-order 5 - point central finite difference scheme in space (4.7) (if the fourth-order accuracy is desired). Here and below, by $L_{h}$ we understand the discrete linear operator obtained using either the second-order approximation to (4.1), or the fourth-order approximation to (4.1), and with $f_{j}$ as the discrete right-hand side.

\section{Second-Order Scheme:}

$$
L_{h}\left[u_{j}\right]:=\frac{1}{h^{2}}\left(k_{j+\frac{1}{2}}\left(u_{j+1}-u_{j}\right)-k_{j-\frac{1}{2}}\left(u_{j}-u_{j-1}\right)\right)-\sigma_{j} u_{j},
$$

the right-hand side $f_{j}:=f\left(x_{j}\right)$, and the coefficients $k_{j+\frac{1}{2}}:=k\left(x_{j+\frac{1}{2}}\right), \sigma_{j}:=\sigma\left(x_{j}\right)$, and $x_{j+\frac{1}{2}}$ is the middle point of the interval $\left[x_{j}, x_{j+1}\right]$.

Fourth - Order Scheme:

$L_{h}\left[u_{j}\right]:=k_{j} \frac{-u_{j-2}+16 u_{j-1}-30 u_{j}+16 u_{j+1}-u_{j+2}}{12 h^{2}}+\left(k_{x}\right)_{j} \frac{u_{j-2}-8 u_{j-1}+8 u_{j+1}-u_{j+2}}{12 h}-\sigma_{j} u_{j}$,

the right-hand side $f_{j}:=f\left(x_{j}\right)$, and the coefficients $k_{j}:=k\left(x_{j}\right),\left(k_{x}\right)_{j}:=k_{x}\left(x_{j}\right), \sigma_{j}:=\sigma\left(x_{j}\right)$. In (4.7), we have used the following fourth-order approximation for

$$
u_{x x} \approx \frac{-u_{j-2}+16 u_{j-1}-30 u_{j}+16 u_{j+1}-u_{j+2}}{12 h^{2}}
$$




$$
u_{x} \approx \frac{u_{j-2}-8 u_{j-1}+8 u_{j+1}-u_{j+2}}{12 h}
$$

Remark: Let us note that the scheme in the form of (4.7) is obtained by rewriting equation $\left(k u_{x}\right)_{x}-\sigma u=f$ in the form of $k u_{x x}+k_{x} u_{x}-\sigma u=f$ (in other words, we assume that this continuous problem (and a nearby problem) is well-posed as well). However, this is not always the best choice, for instance due to some properties of the coefficient $k$, or due to the physics of the problems. In some cases, it is better to discretize the model $\left(k u_{x}\right)_{x}-\sigma u=f$ directly (as we did in (4.6) for the second-order scheme). However, the main ideas of the DPM presented below will not change. Here, we illustrate the ideas using scheme (4.7) for the construction of the fourth-order method, especially since we develop a multi-domain approach in Section 5 (also, the same scheme applies to an equation of the form $k_{1} u_{x x}+k_{2} u_{x}-\sigma u=f$ ). If needed, derivatives of the coefficients, like $k_{x}, \ldots$, can be evaluated by the appropriate finite difference schemes to avoid analytic differentiation.

In general, the linear system of difference equations (4.5) will have multiple solutions since we did not impose any discrete boundary conditions. Once we complete the system (4.5) with the appropriate choice of the numerical boundary conditions, the scheme will result in an accurate approximation of the continuous problem in domain $I$. To do so here, we will develop an approach based on the idea of the Difference Potentials [22, 24].

General Discrete Auxiliary Problem:

One of the major steps of the DPM is the introduction of the auxiliary problem, which we will denote as (AP) and will give definition below.

Definition 4.1. For the given grid function $q \in M^{0}$, find the solution $v \in N^{0}$ of the discrete (AP) such that it satisfies the following system of equations:

$$
\begin{aligned}
L_{h}\left[v_{j}\right] & =q_{j}, \quad x_{j} \in M^{0}, \\
v_{j} & =0, \quad x_{j} \in N^{0} \backslash M^{0} .
\end{aligned}
$$

Here, $L_{h}$ is the same linear discrete operator as in (4.5), but now it is defined on the larger auxiliary domain $\overline{I^{0}}$ (note that, we assumed before in Section 4 that the operator on the left-hand side of the equation (4.1) is well-defined on the entire domain $\overline{I^{0}}$ ). It is applied in (4.10) to the function $v \in N^{0}$. We note that (for small enough $h$ (for (4.7)) and under the above assumptions on the continuous problem) the (AP) (4.10) - (4.11) is well defined for any right hand side $q_{j}$ : it has a unique solution $v \in N^{0}$. In this work we supplemented the discrete (AP) (4.10) by the zero boundary conditions (4.11). In general, the boundary conditions for $(A P)$ are selected to guarantee that the discrete equation $L_{h}\left[v_{j}\right]=q_{j}$ has a unique solution $v \in N^{0}$ for any discrete right-hand side $q$.

Remark: The solution of the (AP) (4.10)-(4.11) defines a discrete Green's operator $G^{h}$ (or the inverse operator to $L_{h}$ ). Although the choice of boundary conditions (4.11) will affect the operator $G^{h}$, and hence the difference potentials and the projections defined below, it will not affect the final approximate solution to (4.1) - (4.2), as long as the (AP) is uniquely 
solvable and well-posed.

\section{Construction of a Particular Solution:}

Let us denote by $u_{j}:=G^{h} f_{j}, u_{j} \in N^{+}$the particular solution of the discrete problem (4.5), which we will construct as the solution (restricted to set $N^{+}$) of the auxiliary problem (AP) (4.10) - (4.11) of the following form:

$$
\begin{aligned}
L_{h}\left[u_{j}\right] & = \begin{cases}f_{j}, & x_{j} \in M^{+}, \\
0, & x_{j} \in M^{-},\end{cases} \\
u_{j} & =0, \quad x_{j} \in N^{0} \backslash M^{0}
\end{aligned}
$$

Remark: The right-hand side of (4.10) in (AP) for the construction of a particular solution, is set to

$$
q_{j}=\left\{\begin{array}{l}
f_{j}, \quad x_{j} \in M^{+} \\
0, \quad x_{j} \in M^{-}
\end{array}\right.
$$

\section{Difference Potential:}

We now introduce a linear space $\mathbf{V}_{\gamma}$ of all the grid functions denoted by $v_{\gamma}$ defined on $\gamma$ $[22],[23,27,4]$, etc. We will extend the value $v_{\gamma}$ by zero to other points of the grid $N^{0}$.

Definition 4.2. The Difference Potential with any given density $v_{\gamma} \in \mathbf{V}_{\gamma}$ is the grid function $u_{j}:=\mathbf{P}_{N^{+}{ }_{\gamma}} v_{\gamma}$, defined on $N^{+}$, and coincides on $N^{+}$with the solution $u_{j}$ of the auxiliary problem (AP) (4.10) - (4.11) of the following form:

$$
\begin{aligned}
L_{h}\left[u_{j}\right] & =\left\{\begin{array}{l}
0, \quad x_{j} \in M^{+}, \\
L_{h}\left[v_{\gamma}\right], \quad x_{j} \in M^{-},
\end{array}\right. \\
u_{j} & =0, \quad x_{j} \in N^{0} \backslash M^{0}
\end{aligned}
$$

Remark: The right-hand side of (4.10) in (AP) for constructing a difference potential with density $v_{\gamma}$ is set to

$$
q_{j}=\left\{\begin{array}{l}
0, \quad x_{j} \in M^{+} \\
L_{h}\left[v_{\gamma}\right], \quad x_{j} \in M^{-},
\end{array}\right.
$$

The Difference Potential with density $v_{\gamma} \in \mathbf{V}_{\gamma}$ is the discrete inverse operator. Here, $\mathbf{P}_{N^{+} \gamma}$ denotes the operator which constructs the difference potential $u_{j}=\mathbf{P}_{N^{+} \gamma} v_{\gamma}$ from the given density $v_{\gamma} \in V_{\gamma}$. The operator $\mathbf{P}_{N^{+} \gamma}$ is the linear operator of the density $v_{\gamma}$. Hence, it can be easily constructed, as illustrated below:

$$
u_{m}=\sum_{j \in \gamma} A_{j m} v_{j}, \quad x_{m} \in N^{+},
$$


with $\sum_{j \in \gamma} A_{j m} v_{j}$ being:

for the second-order method:

$$
\sum_{j \in \gamma} A_{j m} v_{j} \equiv A_{l m} v_{l}+A_{l+1 m} v_{l+1}+A_{L m} v_{L}+A_{L+1 m} v_{L+1}, \quad x_{m} \in N^{+}
$$

and for the fourth-order method:

$$
\begin{aligned}
\sum_{j \in \gamma} A_{j m} v_{j} & \equiv A_{l m} v_{l}+A_{l+1 m} v_{l+1}+A_{l+2 m} v_{l+2}+A_{l+3 m} v_{l+3} \\
& +A_{L m} v_{L}+A_{L+1 m} v_{L+1}+A_{L+2 m} v_{L+2}+A_{L+3 m} v_{L+3}, \quad x_{m} \in N^{+}
\end{aligned}
$$

Here, by $u_{m}$ we denote the value at the grid point $x_{m}$ of the Difference Potential $P_{N_{\gamma}^{+}} v_{\gamma}$ with the density $v_{\gamma}$, and by $\left\{A_{j m}\right\}$ the coefficients of the difference potentials operator. The coefficients $\left\{A_{j m}\right\}$ can be computed by solving an auxiliary problem (AP) (4.15) - (4.16) (or by constructing a Difference Potential operator) with the unit density $v_{\gamma}$ at points $x_{j^{\star}} \in \gamma$. Here, for the second-order method, $x_{j^{\star}} \in \gamma \equiv\left\{x_{l}, x_{l+1}, x_{L}, x_{L+1}\right\}$, and for the fourth-order method, $x_{j^{\star}} \in \gamma \equiv\left\{x_{l}, x_{l+1}, x_{l+2}, x_{l+3}, x_{L}, x_{L+1}, x_{L+2}, x_{L+3}\right\}$. Density $v_{\gamma}$ is defined as the unit density at point $x_{j^{\star}} \in \gamma$ :

$$
v_{\gamma}=\left\{\begin{array}{l}
1, \quad \text { if } j=j^{\star} \\
0, \quad \forall j \neq j^{\star}
\end{array}\right.
$$

Therefore, $A_{j m}$ is the value at a point $x_{m} \in N^{+}$of the solution of the auxiliary problem (AP) (4.15) - (4.16) with the unit density (or the value at a point $x_{m} \in N^{+}$of the Difference Potential with the unit density (4.20)).

Next, similarly to ([22], [3], etc) we can define another operator $\mathbf{P}_{\gamma}: V_{\gamma} \rightarrow V_{\gamma}$ that is defined as the trace (or restriction/projection) of the Difference Potential $\mathbf{P}_{N^{+} \gamma} v_{\gamma}$ on the grid boundary $\gamma$ :

$$
\mathbf{P}_{\gamma} v_{\gamma}:=\operatorname{Tr}_{\gamma}\left(\mathbf{P}_{N^{+} \gamma} v_{\gamma}\right)=\left.\left(\mathbf{P}_{N^{+} \gamma} v_{\gamma}\right)\right|_{\gamma}
$$

We will now formulate the crucial theorem of the method (see [22] for the general result).

Theorem 4.3. Density $u_{\gamma}$ is the trace of some solution $u$ to the Difference Equations (4.5): $u_{\gamma} \equiv T r_{\gamma} u$, if and only if, the following equality holds

$$
u_{\gamma}=\mathbf{P}_{\gamma} u_{\gamma}+G^{h} f_{\gamma},
$$

where $G^{h} f_{\gamma}:=\operatorname{Tr}_{\gamma}\left(G^{h} f\right)$ is the trace (or restriction) of the particular solution $G^{h} f \in N^{+}$ constructed in (4.12) - (4.13) on the grid boundary $\gamma$.

Proof: The proof follows the argument from [22] and for the reader's convenience we will present it below.

First, let us assume that $u_{\gamma}$ is the trace of some solution to the difference equations (4.5): $u_{\gamma}=\operatorname{Tr}_{\gamma} u$, where $u \in N^{+}$is the solution to the difference equations $L_{h}\left[u_{j}\right]=f_{j}, \quad x_{j} \in M^{+}$. 
Construct the grid function: $w:=\mathbf{P}_{N^{+} \gamma} u_{\gamma}+G^{h} f$ on $N^{0}$ (not restricted to $N^{+}$). From the definition of the difference potentials $\mathbf{P}_{N^{+} \gamma} u_{\gamma}$ (4.15), and the particular solution $G^{h} f$ (see (4.12) -(4.13)), the grid function $w \in N^{0}$ coincides with the solution of (AP) (4.10) - (4.11) of the form:

$$
\begin{aligned}
L_{h}\left[w_{j}\right] & =\left\{\begin{array}{l}
f_{j}, \quad x_{j} \in M^{+}, \\
L_{h}\left[u_{\gamma}\right], \quad x_{j} \in M^{-},
\end{array}\right. \\
w_{j} & =0, \quad x_{j} \in N^{0} \backslash M^{0}
\end{aligned}
$$

At the same time, $u \in N^{+}$is the solution of $L_{h}\left[u_{j}\right]=f_{j}, \quad x_{j} \in M^{+}$, hence $f_{j} \equiv L_{h}\left[u_{j}\right]$ in (4.23), and $u_{\gamma}$ is the trace of the solution $u$. Hence we have that:

$$
L_{h}\left[w_{j}\right]=L_{h}\left[u_{j}\right], \quad x_{j} \in M^{0}
$$

Note that solution $u$ is extended by 0 to the points of the set $N^{0} \backslash N^{+}$. Due to the uniqueness argument, $w \equiv u$, on $N^{+}$. Hence, we can reconstruct solution $u$ to the difference equations (4.5) using the formula: $u=\mathbf{P}_{N^{+} \gamma} u_{\gamma}+G^{h} f$. Let us apply the trace operator to both sides of this formula to obtain the desired equality: $u_{\gamma}=\mathbf{P}_{\gamma} u_{\gamma}+G^{h} f_{\gamma}$.

Next, assume that the equality (4.22) holds true for some grid function $u_{\gamma} \in \mathbf{V}_{\gamma}$. Again, let us construct the grid function: $w:=\mathbf{P}_{N^{+} \gamma} u_{\gamma}+G^{h} f$ on $N^{0}$. Thus, $w$ is the solution to (AP) (4.23) - (4.24), and therefore it coincides on $M^{+}$with a solution $u$ of the difference equations (4.5): $w \equiv u$ on $M^{+}$. Hence, due to equality (4.22), $u_{\gamma}$ coincides with the trace $w_{\gamma}$ of $w$, and thus coincides with the trace $u_{\gamma}$ of a solution $u$ of the difference equations (4.5): $u_{\gamma} \equiv T r_{\gamma} u$ (note that for any density $u_{\gamma} \in \mathbf{V}_{\gamma}$, grid function $\mathbf{P}_{N^{+} \gamma} u_{\gamma}+G^{h} f \in N^{+}$is some solution to the difference equations (4.5)).

Remark: Note that the difference potential $\mathbf{P}_{N^{+} \gamma} u_{\gamma}$ is the solution to the homogeneous difference equation $L_{h}\left[u_{j}\right]=0, x_{j} \in M^{+}$, and is uniquely defined once we know the value of the density $u_{\gamma}$ at the points of the boundary $\gamma$.

Also, note that density $u_{\gamma}$ has to satisfy Boundary Equations $u_{\gamma}-P_{\gamma} u_{\gamma}=G^{h} f_{\gamma}$ in order to be a trace of the solution to the difference equation $L_{h}\left[u_{j}\right]=f_{j}$.

Remark: In the case of a constant coefficient model problem (4.1) (assume, $k(x) \equiv 1$ ), using the technique from [21] let us show a direct connection of the difference potential $\mathbf{P}_{N^{+} \gamma} u_{\gamma}$ to the Cauchy-type integral (see $[22,24]$ for more general discussion on the subject). We also assume $\sigma(x)=0$ for simplicity of illustration and will consider the example of the second-order method here (4.6) (for reader's convenience we present similar calculations for the fourth-order method (4.7) in the Appendix Section 8).

Thus, the homogeneous difference equation $L_{h}\left[u_{j}\right]=0, \quad j=l+1, \ldots, L$ for the second order scheme is

$$
\frac{u_{j-1}-2 u_{j}+u_{j+1}}{h^{2}}=0, \quad j=l+1, \ldots, L
$$

Consider a difference equation of the form

$$
z_{j-1}-2 z_{j}+z_{j+1}=0
$$


Denote, $z:=\left(z_{l}, z_{l+1}, . ., z_{L}, z_{L+1}\right)$ to be a solution of the difference equation (4.27). Next, define the generating polynomial

$$
Z(g)=\sum_{j=l}^{L+1} z_{j} g^{j}
$$

where the coefficients of the polynomial $z_{j}$ are the values of the solution $z$ at the grid points. Multiply (4.27) by $g^{j}$, and sum from $l+1, \ldots, L$, thus we will have:

$$
\sum_{j=l+1}^{L} z_{j-1} g^{j}-2 \sum_{j=l+1}^{L} z_{j} g^{j}+\sum_{j=l+1}^{L} z_{j+1} g^{j}=0
$$

For example, we can rewrite the first term as:

$$
g \sum_{j=l+1}^{L} z_{j-1} g^{j-1}=g Z(g)-z_{L} g^{L+1}-z_{L+1} g^{L+2} .
$$

Similarly, the second and the third term in (4.28) can be rewritten as

$$
\sum_{j=l+1}^{L} z_{j} g^{j}=Z(g)-z_{l} g^{l}-z_{L+1} g^{L+1}
$$

and

$$
\frac{1}{g} \sum_{j=l+1}^{L} z_{j+1} g^{j+1}=\frac{1}{g} Z(g)-z_{l} g^{l-1}-z_{l+1} g^{l} .
$$

This way, (4.27) becomes

$$
\left(g Z(g)-z_{L} g^{L+1}-z_{L+1} g^{L+2}\right)-2\left(Z(g)-z_{l} g^{l}-z_{L+1} g^{L+1}\right)+\left(\frac{Z(g)}{g}-z_{l} g^{l-1}-z_{l+1} g^{l}\right)=0 .
$$

Finally, let us recall Cauchy's residue Theorem, and represent

$$
z_{j}=\frac{1}{2 \pi i} \oint_{|g|=2} \frac{Z(g)}{g^{j+1}} d g
$$

Solving (4.29) for $Z(g)$ we obtain

$$
z_{j}=\frac{1}{2 \pi i} \oint_{|g|=2} \frac{g^{l}(1-2 g) z_{l}+g^{l+1} z_{l+1}+g^{L+2} z_{L}+(g-2) g^{L+2} z_{L+1}}{(1-g)^{2} g^{j+1}} d g, \quad j=l, \ldots, L+1
$$

The Cauchy - type integral (4.30) plays the role of the discrete potential for the linear difference equations (4.27) (each $z_{j}, j=l, \ldots, L+1$ is determined by values $z_{\gamma}$ ), as the difference potential $\mathbf{P}_{N^{+} \gamma} u_{\gamma}$ for the linear difference equations (4.26).

Coupling of Boundary Equations with Boundary Conditions: 
We will present below the details for the second-order scheme and for the fourth-order scheme separately since there are differences in the technical details (however, the main strategy is the same for any high-order scheme).

\section{Case of the Second-Order Method:}

The Boundary Equations: $u_{\gamma}-P_{\gamma} u_{\gamma}=G^{h} f_{\gamma}$ for the unknown density $u_{\gamma}$ is the linear system of equations:

$$
(I-A) \mathbf{u}=\mathbf{G}^{\mathbf{h}} \mathbf{f},
$$

where $I$ is the identity matrix and $A$ is the matrix of the coefficients of the difference potentials with unit densities:

$$
\left(\begin{array}{cccc}
A_{l l} & A_{l+1 l} & A_{L l} & A_{L+1 l} \\
A_{l l+1} & A_{l+1 l+1} & A_{L l+1} & A_{L+1 l+1} \\
A_{l L} & A_{l+1 L} & A_{L L} & A_{L+1 L} \\
A_{l L+1} & A_{l+1 L+1} & A_{L L+1} & A_{L+1 L+1}
\end{array}\right)
$$

The column vector of the unknown densities is

$$
\mathbf{u}:=\left(u_{l}, u_{l+1}, u_{L}, u_{L+1}\right)^{T},
$$

and the column vector of the right-hand side is

$$
\mathbf{G}^{\mathbf{h}} \mathbf{f}:=\left(G^{h} f_{l}, G^{h} f_{l+1}, G^{h} f_{L}, G^{h} f_{L+1}\right)^{T} .
$$

The above system of Boundary Equations (4.31) will have multiple solutions without boundary conditions (4.2), since it is equivalent to the difference equations $L_{h}\left[u_{j}\right]=f_{j}, x_{j} \in M^{+}$. We need to supplement it by the boundary conditions (4.2) to construct the unique $u_{\gamma}$.

Remark: It can be shown (see for example, [22] or [3]) that the rank of the linear system will be $\left|\gamma^{i n}\right|$ (here, $\left|\gamma^{i n}\right|$ is the cardinality of a set $\gamma^{i n}$ - the interior layer of the grid boundary $\gamma=\gamma^{i n} \cup \gamma^{e x}$. Similarly, $\gamma^{e x}$ denotes the exterior layer). For the second order method here, the rank is 2 .

Therefore, we will consider the following approach to solve for the unknown densities $u_{\gamma}$ from the Boundary Equations (4.31). Here, using the idea of the Taylor expansion, one can represent the unknown densities $u_{\gamma}$ with the values of the continuous solution and its derivatives at the boundary of the domain with the desired accuracy: in other words, one can define the extension operator from the continuous boundary $\partial I$ to the discrete boundary $\gamma$ for the solution of (4.1). Note that the extension operator (the way it is constructed below) depends only on the properties of the given model at the continuous boundary $\partial I$. For example, in case of 3 terms, the extension operator is:

$$
u_{j}:=\left.u\right|_{\partial I} \pm\left. d u_{x}\right|_{\partial I}+\left.\frac{d^{2}}{2} u_{x x}\right|_{\partial I}, \quad x_{j} \in \gamma
$$

where

$$
\left.u\right|_{\partial I}:=u(0),\left.u_{x}\right|_{\partial I}:=u_{x}(0),\left.u_{x x}\right|_{\partial I}:=u_{x x}(0), \text { if } j=\{l, l+1\},
$$


and

$$
\left.u\right|_{\partial I}:=u(1),\left.u_{x}\right|_{\partial I}:=u_{x}(1),\left.u_{x x}\right|_{\partial I}:=u_{x x}(1), \text { if } j=\{L, L+1\} .
$$

$d$ denotes the distance from point $x_{j} \in \gamma$ to the boundary point. We take it with either sign "+" or with sign "-".

The value $\left.u\right|_{\partial I}$ is given due to the boundary conditions (4.2). Let us denote the unknown value of $C_{1}:=u_{x}(0)$ and $C_{2}:=u_{x}(1)$. We can obtain the values of higher-order derivatives using the given differential equation (4.1). In case of the second-order derivatives, this is simply

$$
u_{x x}(0)=\frac{f(0)+\sigma(0) a}{k(0)}-\frac{k_{x}(0)}{k(0)} C_{1},
$$

and

$$
u_{x x}(1)=\frac{f(1)+\sigma(1) b}{k(1)}-\frac{k_{x}(1)}{k(1)} C_{2}
$$

Hence, the only unknowns that we need to solve for are $C_{1}$ and $C_{2}$. We will use expansion (4.32) for $u_{\gamma}$ in the boundary equations (4.31) and obtain the overdetermined linear system for $C_{1}$ and $C_{2}$. This system is solved uniquely using the least square method. After that, we can obtain the value of the density $u_{\gamma}$ at the points of the grid boundary $\gamma$ using formula (4.32).

\section{Case of the Fourth-Order Method:}

Similarly to the second-order case above, the Boundary Equations: $u_{\gamma}-P_{\gamma} u_{\gamma}=G^{h} f_{\gamma}$ for the unknown density $u_{\gamma}$ is the linear system of equations:

$$
(I-A) \mathbf{u}=\mathbf{G}^{\mathbf{h}} \mathbf{f},
$$

where $I$ is the identity matrix and $A$ is the matrix of the coefficients of the difference potentials with unit densities:

$$
\left(\begin{array}{cccccccc}
A_{l l} & A_{l+1 l} & A_{l+2 l} & A_{l+3 l} & A_{L l} & A_{L+1 l} & A_{L+2 l} & A_{L+3 l} \\
A_{l l+1} & A_{l+1 l+1} & A_{l+2 l+1} & A_{l+3 l+1} & A_{L l+1} & A_{L+1 l+1} & A_{L+2 l+1} & A_{L+3 l+1} \\
A_{l l+2} & A_{l+1 l+2} & A_{l+2 l+2} & A_{l+3 l+2} & A_{L l+2} & A_{L+1 l+2} & A_{L+2 l+2} & A_{L+3 l+2} \\
A_{l l+3} & A_{l+1 l+3} & A_{l+2 l+3} & A_{l+3 l+3} & A_{L l+3} & A_{L+1 l+3} & A_{L+2 l+3} & A_{L+3 l+3} \\
A_{l L} & A_{l+1 L} & A_{l+2 L} & A_{l+3 L} & A_{L L} & A_{L+1 L} & A_{L+2 L} & A_{L+3 L} \\
A_{l L+1} & A_{l+1 L+1} & A_{l+2 L+1} & A_{l+3 L+1} & A_{L L+1} & A_{L+1 L+1} & A_{L+2 L+1} & A_{L+3 L+1} \\
A_{l L+2} & A_{l+1 L+2} & A_{l+2 L+2} & A_{l+3 L+2} & A_{L L+2} & A_{L+1 L+2} & A_{L+2 L+2} & A_{L+3 L+2} \\
A_{l L+3} & A_{l+1 L+3} & A_{l+2 L+3} & A_{l+3 L+3} & A_{L L+3} & A_{L+1 L+3} & A_{L+2 L+3} & A_{L+3 L+3}
\end{array}\right)
$$

The column vector of the unknown densities is

$$
\mathbf{u}:=\left(u_{l}, u_{l+1}, u_{l+2}, u_{l+3}, u_{L}, u_{L+1}, u_{L+2}, u_{L+3}\right)^{T},
$$

and the column vector of the right-hand side is

$$
\mathbf{G}^{\mathbf{h}} \mathbf{f}:=\left(G^{h} f_{l}, G^{h} f_{l+1}, G^{h} f_{l+2}, G^{h} f_{l+3}, G^{h} f_{L}, G^{h} f_{L+1}, G^{h} f_{L+2}, G^{h} f_{L+3}\right)^{T} .
$$


As before, the above system of Boundary Equations (4.35) without boundary conditions (4.2) will have multiple solutions, since it is equivalent to the difference equations $L_{h}\left[u_{j}\right]=f_{j}, x_{j} \in$ $M^{+}$. To construct the unique $u_{\gamma}$ we need to supplement it by the boundary conditions (4.2).

Remark: The rank of the system here is 4 for the fourth order scheme.

Therefore, similarly to the second-order case, we will consider the following approach to solve for the unknown densities $u_{\gamma}$ from the Boundary Equations (4.35). Again, using the idea of Taylor expansion, we will construct the extension from the continuous boundary $\partial I$ to the discrete boundary $\gamma$ of the solution to (4.1) For example, in case of 5-terms, extension operator is:

$$
u_{j}:=\left.u\right|_{\partial I} \pm\left. d u_{x}\right|_{\partial I}+\left.\frac{d^{2}}{2 !} u_{x x}\right|_{\partial I} \pm\left.\frac{d^{3}}{3 !} u_{x x x}\right|_{\partial I}+\left.\frac{d^{4}}{4 !} u_{x x x x}\right|_{\partial I} \quad x_{j} \in \gamma
$$

where, if $j=\{l, l+1, l+2, l+3\}$, we have that:

$$
\left.u\right|_{\partial I}:=u(0),\left.u_{x}\right|_{\partial I}:=u_{x}(0),\left.u_{x x}\right|_{\partial I}:=u_{x x}(0),\left.u_{x x x}\right|_{\partial I}:=u_{x x x}(0),\left.u_{x x x x}\right|_{\partial I}:=u_{x x x x}(0),
$$

and if $j=\{L, L+1, L+2, L+3\}$, we denote:

$$
\left.u\right|_{\partial I}:=u(1),\left.u_{x}\right|_{\partial I}:=u_{x}(1),\left.u_{x x}\right|_{\partial I}:=u_{x x}(1),\left.u_{x x x}\right|_{\partial I}:=u_{x x x}(1),\left.u_{x x x x}\right|_{\partial I}:=u_{x x x x}(1) .
$$

$d$ is the distance from point $x_{j} \in \gamma$ to the boundary point. We take it with either sign "+" or sign "-".

$\left.u\right|_{\partial I}$ are given due to the boundary conditions (4.2). Let us denote the unknown value of $C_{1}:=u_{x}(0)$ and $C_{2}:=u_{x}(1)$. We can obtain the values of higher-order derivatives using the given differential equation (4.1). In case of the second-order derivatives, this is simply

$$
u_{x x}(0)=\frac{f(0)+\sigma(0) a}{k(0)}-\frac{k_{x}(0)}{k(0)} C_{1},
$$

and

$$
u_{x x}(1)=\frac{f(1)+\sigma(1) b}{k(1)}-\frac{k_{x}(1)}{k(1)} C_{2}
$$

For the third order derivatives we have:

$$
\begin{aligned}
& u_{x x x}(0)=\frac{f_{x}(0)-\frac{2 k_{x}(0)}{k(0)} f(0)}{k(0)}+\frac{\sigma_{x}(0)-\frac{2 k_{x}(0)}{k(0)} \sigma(0)}{k(0)} a+\frac{\sigma(0)+2 \frac{k_{x}^{2}(0)}{k(0)}-k_{x x}(0)}{k(0)} C_{1} \\
& u_{x x x}(1)=\frac{f_{x}(1)-\frac{2 k_{x}(1)}{k(1)} f(1)}{k(1)}+\frac{\sigma_{x}(1)-\frac{2 k_{x}(1)}{k(1)} \sigma(1)}{k(1)} b+\frac{\sigma(1)+2 \frac{k_{x}^{2}(1)}{k(1)}-k_{x x}(1)}{k(1)} C_{2}
\end{aligned}
$$


And for the fourth order derivatives we have:

$$
\begin{aligned}
u_{x x x x}(0) & =-\frac{3 k_{x x}(0)-\frac{6 k_{x}^{2}(0)}{k(0)}-\sigma(0)}{k^{2}(0)} f(0)-\frac{3 k_{x}(0)}{k^{2}(0)} f_{x}(0)+\frac{f_{x x}(0)}{k(0)} \\
& -\left(\frac{3 k_{x x}(0)-\sigma(0)}{k^{2}(0)} \sigma(0)+\frac{3 k_{x}(0)\left(\sigma_{x}(0)-\frac{2 k_{x}(0)}{k(0)} \sigma(0)\right)}{k^{2}(0)}-\frac{\sigma_{x x}(0)}{k(0)}\right) a \\
& +\left(\frac{3 k_{x x}(0)-\sigma(0)}{k^{2}(0)} k_{x}(0)-3 k_{x} \frac{\sigma(0)+2 \frac{k_{x}^{2}(0)}{k(0)}-k_{x x}(0)}{k^{2}(0)}-\frac{k_{x x x}(0)-2 \sigma_{x}(0)}{k(0)}\right) C_{1} \\
u_{x x x x}(1) & =-\frac{3 k_{x x}(1)-\frac{6 k_{x}^{2}(1)}{k(1)}-\sigma(1)}{k^{2}(1)} f(1)-\frac{3 k_{x}(1)}{k^{2}(1)} f_{x}(1)+\frac{f_{x x}(1)}{k(1)} \\
& -\left(\frac{3 k_{x x}(1)-\sigma(1)}{k^{2}(1)} \sigma(1)+\frac{3 k_{x}(1)\left(\sigma_{x}(1)-\frac{2 k_{x}(1)}{k(1)} \sigma(1)\right)}{k^{2}(1)}-\frac{\sigma_{x x}(1)}{k(1)}\right) b \\
& +\left(\frac{3 k_{x x}(1)-\sigma(1)}{k^{2}(1)} k_{x}(1)-3 k_{x} \frac{\sigma(1)+2 \frac{k_{x}^{2}(1)}{k(1)}-k_{x x}(1)}{k^{2}(1)}-\frac{k_{x x x}(1)-2 \sigma_{x}(1)}{k(1)}\right) C_{2}
\end{aligned}
$$

Hence, again, the only unknowns that we need to solve for are $C_{1}$ and $C_{2}$. We will use expansion (4.36) for $u_{\gamma}$ in the boundary equations (4.35), and obtain the overdetermined linear system for $C_{1}$ and $C_{2}$. This system is solved uniquely for $C_{1}$ and $C_{2}$ using the least square method. After that, we can obtain the value of the density $u_{\gamma}$ at the points of the grid boundary $\gamma$ using formula (4.36).

Finally, the last step of the DPM is to use the obtained density $u_{\gamma}$ to reconstruct the approximation to the solution (4.1) - (4.2) inside the domain I.

Generalized Green's Formula:

Theorem 4.4. Discrete solution $u_{j}:=\mathbf{P}_{N^{+}{ }_{\gamma}} u_{\gamma}+G^{h} f$ is the approximation to the solution $u_{j} \approx u\left(x_{j}\right), x_{j} \in N^{+} \cap I$ of the continuous problem (4.1) - (4.2).

Discussion: The result is the consequence of the sufficient regularity (smoothness) of the exact solution, Theorem 4.3, extension operator (4.32) (for the second-order method) or the extension operator (4.36) (for the fourth-order method), and the second-order accuracy of the scheme (4.6) (for the second-order method) and the fourth-order accuracy of the scheme (4.7) (for the fourth-order method). Therefore, we expect that the discrete solution $u_{j}:=\mathbf{P}_{N^{+} \gamma} u_{\gamma}+G^{h} f$ will approximate the solution $u_{j} \approx u\left(x_{j}\right), x_{j} \in N^{+} \cap I$ of the continuous problem (4.1) - (4.2) with $O\left(h^{2}\right)$ (for the second-order method) and with $O\left(h^{4}\right)$ (for the fourth-order method) in the maximum norm. In Section 6 we illustrate the capabilities and the consistence of the developed approach with several numerical experiments for the interface/composite domain problems. 
Let us remark, that in higher-dimensions $(\geq 2)$, in $[19,20]$ it was shown (under sufficient regularity of the exact solution), that the Difference Potentials approximate surface potentials of the elliptic operators (and, hence DPM approximates the solution to the elliptic boundary value problem) with the accuracy of $O\left(h^{\mathcal{P}-\varepsilon}\right)$ in the discrete Hölder norm of order $\mathcal{Q}+\varepsilon$. Here, $0<\varepsilon<1$ is arbitrary number, $\mathcal{Q}$ is the order of the considered elliptic operator, and $\mathcal{P}=2$ - if the second-order scheme is employed for the approximation of the elliptic operator, or $\mathcal{P}=4$ - if the fourth-order scheme is employed for the approximation of the elliptic operator (see [19, 20] or [22] for the details and proof of the general result. Also, see [16] for the brief discussion of the accuracy of DPM). However, the rigorous theoretical analysis (accuracy, etc) of more general concept of the Difference Potentials for arbitrary linear difference scheme still needs to be investigated [24].

\section{Remark:}

- The formula $\mathbf{P}_{N^{+} \gamma} u_{\gamma}+G^{h} f$ is known as the discrete generalized Green's formula.

- Note that after density $u_{\gamma}$ is obtained from the Boundary Equations, the difference potential is easily constructed as the solution of a simple (AP) using Def. 4.2.

\section{Difference potentials approach for interface and composite domains problems}

In Section 4.1 we formulated second and fourth-order methods based on Difference Potentials approach, for problems in the single domain $I$. In this section we will show how to extend these methods to interface/composite domains problems (3.1) - (3.4).

First, as we have done in Section 4 for the single domain $I$, we will introduce the auxiliary domains. We will place each of the original subdomains $I_{s}$ in the auxiliary domains $I_{s}^{0} \subset$ $\mathbb{R},(s=1,2)$ and will formulate the auxiliary difference problems in each subdomain $I_{s},(s=$ $1,2)$. The choice of these auxiliary domains $I_{1}^{0}$ and $I_{2}^{0}$ does not need to depend on each other. Again, for each subdomain, we will proceed in a similar way as we did in Section 4.1. Also, for each $I_{s}^{0}$ we will introduce, for example a Cartesian grid (the choice of the grids for the auxiliary problems in each subdomain will be independent. The choice for each subdomain is based on the considerations of the properties of the model and solution in each subdomain (3.1) - (3.3), as well as the efficiency and simplicity of the resulting discrete problems). After that, all the definitions, notations, and properties introduced in Section 4.1 extend to each subdomain $I_{s}$ in a direct and straightforward way: we will use index $s,(s=1,2)$ to distinguish each subdomain. Let us denote the difference problem of $(3.1)$ (3.2) for each subdomain as:

$$
L_{h}^{s}\left[u_{j}\right]=f_{s j}, \quad x_{j} \in M_{s}^{+},
$$

The difference problem (5.1) is obtained using either the second-order (4.6) or the fourthorder scheme (4.7). 
The cornerstone of our approach for the composite domains and interface problems is the following proposition.

Theorem 5.1. Density $u_{\gamma}:=\left(u_{\gamma_{1}}, u_{\gamma_{2}}\right)$ is the trace of some solution $u \in I_{1} \cup I_{2}$ to the Difference Equations (5.1): $u_{\gamma} \equiv T r_{\gamma} u$, if and only if, the following equality holds

$$
\begin{aligned}
& u_{\gamma_{1}}=\mathbf{P}_{\gamma_{1}} u_{\gamma_{1}}+G^{h} f_{\gamma_{1}}, x_{j} \in \gamma_{1} \\
& u_{\gamma_{2}}=\mathbf{P}_{\gamma_{2}} u_{\gamma_{2}}+G^{h} f_{\gamma_{2}}, x_{j} \in \gamma_{2}
\end{aligned}
$$

The obtained discrete solution $u_{j}:=\mathbf{P}_{\mathbf{s}_{N_{s}}^{+} \gamma_{s}} u_{\gamma_{s}}+G_{s}^{h} f_{s}$ is the approximation to the solution $u_{j} \approx u\left(x_{j}\right) \in I_{1} \cup I_{2}, x_{j} \in N_{s}^{+} \cap I_{s}, s=1,2$ of the continuous problem (3.1) - (3.4).

Discussion: The result is a consequence of the results in Section 4.1. We expect that the solution $u_{j}:=\mathbf{P}_{\mathbf{s}_{N_{s}}^{+} \gamma_{s}} u_{\gamma_{s}}+G_{s}^{h} f_{s}$ will approximate the exact solution $u\left(x_{j}\right) \in I_{1} \cup I_{2}$, $x_{j} \in N_{s}^{+} \cap I_{s}, s=1,2$ with the accuracy $O\left(h^{2}\right)$ for the second - order scheme, and with the accuracy $O\left(h^{4}\right)$ for the fourth - order scheme in the maximum norm. See also Section 6 for the numerical results.

Remark: Similar to the discussion in Section 4.1, the Boundary Equations (5.2) -(5.3) alone will have multiple solutions and have to be coupled with boundary (3.3) and interface conditions (3.4) to obtain the unique densities $u_{\gamma_{1}}$ and $u_{\gamma_{2}}$. We use the extension formula (4.32) (second-order scheme) or (4.36) (fourth-order scheme) to construct $u_{\gamma_{s}}, s=1,2$ in each subdomain/domain. The unknowns are $\left.u\right|_{\partial I_{1}},\left.u_{x}\right|_{\partial I_{1}}$ and $\left.u\right|_{\partial I_{2}},\left.u_{x}\right|_{\partial I_{2}}$. Here, $\partial I_{1}:=\{0, \alpha\}$ and $\partial I_{2}:=\{\alpha, 1\}$ (total 8 unknowns without imposed boundary and interface conditions (3.3) $-(3.4))$.

Note that we constructed the algorithm here based on the inhomogeneous Boundary Equations (5.2) - (5.3) instead of the homogenous Boundary Equations $u_{\gamma_{s}}-\mathbf{P}_{\mathbf{s} \gamma_{s}} u_{\gamma_{s}}=0$ like in $[23,27,4]$. We do not see too many advantages of one approach over the other one in $1 D$, but in $2 D$ and in $3 D$ we expect that the algorithms based on homogenous Boundary Equations will be more efficient and will have more flexibility, such as the ability to consider different auxiliary problems for the construction of the difference potentials and the particular solutions, etc. (see for more details in our work [23, 27, 4]). This will be part of our future research for problems with variable coefficients in $2 D$ and in $3 D$.

\section{Numerical examples}

In this section, we will consider two test problems. We will first compare the performance of the second-order Difference Potentials Method (DPM) with the second-order Immersed Interface Method (IIM) [10, 9, 11], as well as with the standard second-order central difference method in Section 6.1. Moreover, we will present the result of the fourth-order DPM for 
the same test problem. Next, in Section 6.2 we will test and compare the second and the fourth-order DPM on the variable coefficient problem in heterogeneous media as well. In all numerical experiments below, we compute the maximum error

$$
\max _{x_{j} \in[0,1]}\left|u\left(x_{j}\right)-u_{j}\right|
$$

Moreover, in Tables 3 - 4 and in Table 8 to further illustrate the potential of the developed approach to capture the discontinuities at the interface, we also compute the maximum error between the discrete gradient (derivative) of the exact solution and the numerical solution

$$
\max _{\left(x_{j+1}, x_{j}\right) \in[0,1]}\left|\frac{u\left(x_{j+1}\right)-u\left(x_{j}\right)}{h}-\frac{u_{j+1}-u_{j}}{h}\right| .
$$

Here, $u\left(x_{j}\right)$ is the exact solution at the grid points, $u_{j}$ is the numerical solution and $h$ is the mesh size.

\subsection{Second and fourth order difference potentials method and comparison with other methods}

To test and compare second and fourth order DPM, second-order IIM and the standard central second-order finite difference method we consider the following problems in this section (which is the modification of a problem in [11]).

$$
\left(\beta u_{x}\right)_{x}=56 x^{6}, \quad \beta=\left\{\begin{array}{lc}
1, & \text { if } 0 \leq x \leq 0.5 \\
2, & \text { if } 0.5<x \leq 1
\end{array}\right.
$$

subject to the boundary and interface conditions:

$$
\begin{aligned}
u(0) \equiv u_{1}(0)=0, & u(1) \equiv u_{2}(1)=\frac{257}{512} \\
u_{1}(0.5) & =u_{2}(0.5), \\
u_{1 x}(0.5) & =2 u_{2 x}(0.5)
\end{aligned}
$$

The exact solution to (6.1) - (6.4) is given as:

$$
u(x)= \begin{cases}u_{1}(x)=x^{8}, & \text { if } x \leq 0.5 \\ u_{2}(x)=\frac{1}{2}\left(x^{8}+\frac{1}{256}\right), & \text { if } x>0.5\end{cases}
$$

In the Tables below, DPM 2 stands for second-order DPM, DPM 4 stands for the fourth-order DPM, and IIM 2 stands for the second-order IIM. For DPM 2 and DPM 4, we implement the algorithm from Section 5. We consider auxiliary domain $[-0.25,0.75]$ in Tables $1-4$, and auxiliary domain $[-0.667,0.833]$ in Tables $5-6$ to discretize the problem using DPM in subdomain $I_{1}:=[0,0.5]$. We consider auxiliary domain $[0.25,1.25]$ in Tables $1-4$, and auxiliary domain $[-0.167,1.33]$ in Tables $5-6$ to discretize the problem using DPM in subdomain $I_{2}:=[0.5,1.0]$. Each auxiliary domain is subdivided by $N$ intervals, and in Tables 1 - 6 we use the same number of intervals (the same grids) for each subdomain. The 
results presented in Tables 1 - 4 show that the errors of the second-order DPM 2 and the second-order IIM 2 are similar (there is difference in $6^{\text {th }}-8^{\text {th }}$ digits when small enough $h$ is considered and we believe that this is due to different effect of round off errors in DPM and IIM). Moreover, the results presented in Tables 3 - 4 show the ability of the Difference Potentials approach to capture very accurately discontinuities at the interface.

We believe that these results are expected. The proposed method here is based on the idea of the difference potentials, which allows to construct Boundary Equations with Projection for the trace of the solution at the points near a continuous boundary (at the points of the discrete grid boundary). Therefore, the accuracy of DPM is only limited by the accuracy of the scheme employed to construct the difference potentials and the particular solutions (see a more detailed exposition of the theory in [22]). Hence, the accuracy of the DPM 2 in this case is limited only by the second-order scheme. At the same time, IIM is derived from the idea of minimizing the magnitude of local truncation error near the irregular points (near interface) using the explicit information about jump conditions on the solution and the flux across the interface. This allows to obtain a numerical scheme that achieves second-order accuracy (for a second-order scheme; note, that extension of IIM to higher than second order is not straightforward) on the interface problems [11]. Thus, the accuracy of the second-order IIM and the accuracy of the second-order DPM is very close to each other. Similar results are observed in 2D when DPM is compared with the second order finite difference scheme $[27,4]$ (when classical solution exists).

At the same time, as expected, and illustrated in Table 7, the standard centered secondorder finite-differences scheme failed to converge on the interface problem (6.1) - (6.2). Furthermore, the results in Tables 1 - 6 confirm second-order convergence for DPM 2 and fourth-order convergence for DPM 4 in the solution, as well as in the discrete derivative of the solution - Tables 3 - 4 (we consider different choice of auxiliary problems for DPM in Tables 1 - 4 and in Tables 5-6). Let us remark that the breakdown of convergence of fourth-order scheme on finer grids is due to the loss of significant digits, as the absolute levels of error get very close to machine zero.

\begin{tabular}{|c|c|c|c|c|c|c|}
\hline $\mathrm{N}$ & Error (DPM 2) & Conv. Rate & Error (IIM 2) & Conv. Rate & Error (DPM 4) & Conv. Rate \\
\hline 20 & 0.003998 & & 0.003998 & & $7.361 \mathrm{e}-05$ & \\
40 & 0.001002 & 1.996 & 0.001002 & 1.996 & $2.346 \mathrm{e}-06$ & 4.972 \\
80 & 0.0002506 & 1.999 & 0.0002506 & 1.999 & $8.688 \mathrm{e}-08$ & 4.755 \\
160 & $6.267 \mathrm{e}-05$ & 2.000 & $6.267 \mathrm{e}-05$ & 2.000 & $6.92 \mathrm{e}-09$ & 3.650 \\
320 & $1.567 \mathrm{e}-05$ & 2.000 & $1.567 \mathrm{e}-05$ & 2.000 & $4.756 \mathrm{e}-10$ & 3.863 \\
640 & $3.917 \mathrm{e}-06$ & 2.000 & $3.917 \mathrm{e}-06$ & 2.000 & $1.119 \mathrm{e}-10$ & 2.088 \\
\hline
\end{tabular}

Table 1: Errors in the solution as functions of the number of intervals: for DPM 2 and DPM 4 we consider auxiliary domains $[-0.25,0.75]$ for $0 \leq x \leq 0.5$, and $[0.25,1.25]$ for $0.5<x \leq 1$. The mesh size $h$ is the same for DPM and IIM due to the choice of the auxiliary domains. Problem (6.1).

Finally, we use the test problem below (6.6) - (6.9) to illustrate that the fourth-order DPM 


\begin{tabular}{|c|c|c|c|c|c|c|}
\hline $\mathrm{N}$ & Error (DPM 2) & Conv. Rate & Error (IIM 2) & Conv. Rate & Error (DPM 4) & Conv. Rate \\
\hline 24 & 0.002775 & & 0.002775 & & $2.996 \mathrm{e}-05$ & \\
48 & 0.000696 & 1.995 & 0.000696 & 1.995 & $9.408 \mathrm{e}-07$ & 4.993 \\
96 & 0.0001741 & 1.999 & 0.0001741 & 1.999 & $4.579 \mathrm{e}-08$ & 4.361 \\
192 & $4.352 \mathrm{e}-05$ & 2.000 & $4.352 \mathrm{e}-05$ & 2.000 & $3.453 \mathrm{e}-09$ & 3.729 \\
384 & $1.088 \mathrm{e}-05$ & 2.000 & $1.088 \mathrm{e}-05$ & 2.000 & $2.141 \mathrm{e}-10$ & 4.012 \\
768 & $2.72 \mathrm{e}-06$ & 2.000 & $2.72 \mathrm{e}-06$ & 2.000 & $2.641 \mathrm{e}-10$ & -0.303 \\
\hline
\end{tabular}

Table 2: Errors in the solution as functions of the number of intervals: for DPM 2 and DPM 4 we consider auxiliary domains $[-0.25,0.75]$ for $0 \leq x \leq 0.5$, and $[0.25,1.25]$ for $0.5<x \leq 1$. The mesh size $h$ is the same for DPM and IIM due to the choice of the auxiliary domains. Problem (6.1).

\begin{tabular}{|c|c|c|c|c|c|c|}
\hline $\mathrm{N}$ & Error (DPM 2) & Conv. Rate & Error (IIM 2) & Conv. Rate & Error (DPM 4) & Conv. Rate \\
\hline 20 & 0.019756 & & 0.019756 & & $4.629 \mathrm{e}-04$ & \\
40 & 0.006241 & 1.663 & 0.006241 & 1.663 & $3.579 \mathrm{e}-05$ & 3.693 \\
80 & 0.001743 & 1.840 & 0.001743 & 1.840 & $2.412 \mathrm{e}-06$ & 3.891 \\
160 & $4.600 \mathrm{e}-04$ & 1.922 & $4.600 \mathrm{e}-04$ & 1.922 & $1.555 \mathrm{e}-07$ & 3.955 \\
320 & $1.181 \mathrm{e}-04$ & 1.961 & $1.181 \mathrm{e}-04$ & 1.961 & $9.846 \mathrm{e}-09$ & 3.981 \\
640 & $2.992 \mathrm{e}-05$ & 1.981 & $2.992 \mathrm{e}-05$ & 1.981 & $4.044 \mathrm{e}-10$ & 4.606 \\
\hline
\end{tabular}

Table 3: Errors in the discrete gradient (derivative) of the solution as functions of the number of intervals: for $D P M 2$ and $D P M 4$ we consider auxiliary domains [-0.25, 0.75] for $0 \leq x \leq 0.5$, and [0.25, 1.25] for $0.5<x \leq 1$. The mesh size $h$ is the same for DPM and IIM due to the choice of the auxiliary domains. Problem (6.1).

\begin{tabular}{|c|c|c|c|c|c|c|}
\hline $\mathrm{N}$ & Error (DPM 2) & Conv. Rate & Error (IIM 2) & Conv. Rate & Error (DPM 4) & Conv. Rate \\
\hline 24 & 0.014861 & & 0.014861 & & $2.421 \mathrm{e}-04$ & \\
48 & 0.004499 & 1.724 & 0.004499 & 1.724 & $1.773 \mathrm{e}-05$ & 3.771 \\
96 & 0.001233 & 1.868 & 0.001233 & 1.868 & $1.176 \mathrm{e}-06$ & 3.915 \\
192 & $3.223 \mathrm{e}-04$ & 1.935 & $3.223 \mathrm{e}-04$ & 1.935 & $7.534 \mathrm{e}-08$ & 3.964 \\
384 & $8.239 \mathrm{e}-05$ & 1.968 & $8.239 \mathrm{e}-05$ & 1.968 & $4.731 \mathrm{e}-09$ & 3.993 \\
768 & $2.083 \mathrm{e}-05$ & 1.984 & $2.083 \mathrm{e}-05$ & 1.984 & $6.565 \mathrm{e}-10$ & 2.849 \\
\hline
\end{tabular}

Table 4: Errors in the discrete gradient (derivative) of the solution as functions of the number of intervals: for DPM 2 and DPM 4 we consider auxiliary domains [-0.25, 0.75] for $0 \leq x \leq 0.5$, and $[0.25,1.25]$ for $0.5<x \leq 1$. The mesh size $h$ is the same for DPM and IIM due to the choice of the auxiliary domains. Problem (6.1). 


\begin{tabular}{|c|c|c|c|c|c|}
\hline $\mathrm{N}$ & Error (DPM 2) & Conv. Rate & $\mathrm{N}$ & Error (DPM 2) & Conv. Rate \\
\hline 20 & 0.009871 & & 24 & 0.006677 & \\
40 & 0.002115 & 2.223 & 48 & 0.001521 & 2.134 \\
80 & 0.0005357 & 1.981 & 96 & 0.0003978 & 1.935 \\
160 & 0.0001488 & 1.848 & 192 & $9.719 \mathrm{e}-05$ & 2.033 \\
320 & $3.441 \mathrm{e}-05$ & 2.113 & 384 & $2.458 \mathrm{e}-05$ & 1.983 \\
640 & $8.59 \mathrm{e}-06$ & 2.002 & 768 & $6.109 \mathrm{e}-06$ & 2.009 \\
\hline
\end{tabular}

Table 5: Errors in the solution as functions of the number of intervals for DPM 2 : we consider auxiliary domains $[-0.667,0.833]$ for $0 \leq x \leq 0.5$, and $[-0.167,1.33]$ for $0.5<x \leq 1$. Problem (6.1).

\begin{tabular}{|c|c|c|c|c|c|}
\hline $\mathrm{N}$ & Error (DPM 4) & Conv. Rate & $\mathrm{N}$ & Error (DPM 4) & Conv. Rate \\
\hline 20 & 0.0001759 & & 24 & $9.473 \mathrm{e}-05$ & \\
40 & $9.466 \mathrm{e}-06$ & 4.216 & 48 & $4.397 \mathrm{e}-06$ & 4.429 \\
80 & $6.124 \mathrm{e}-07$ & 3.950 & 96 & $2.746 \mathrm{e}-07$ & 4.001 \\
160 & $4.202 \mathrm{e}-08$ & 3.865 & 192 & $1.972 \mathrm{e}-08$ & 3.800 \\
320 & $2.361 \mathrm{e}-09$ & 4.154 & 384 & $1.231 \mathrm{e}-09$ & 4.002 \\
640 & $1.117 \mathrm{e}-10$ & 4.402 & 768 & $1.286 \mathrm{e}-10$ & 3.259 \\
\hline
\end{tabular}

Table 6: Errors in the solution as functions of the number of intervals for DPM 4: we consider auxiliary domains [-0.667, 0.833] for $0 \leq x \leq 0.5$, and [-0.167,1.33] for $0.5<x \leq 1$. Problem (6.1).

\begin{tabular}{|c|c|c|c|}
\hline $\mathrm{N}$ & Error (Standard Central FD) & $\mathrm{N}$ & Error (Standard Central FD) \\
\hline 20 & 0.006789 & 24 & 0.007191 \\
40 & 0.009214 & 48 & 0.009633 \\
80 & 0.01037 & 96 & 0.01053 \\
160 & 0.01083 & 192 & 0.01089 \\
320 & 0.01103 & 384 & 0.01106 \\
640 & 0.01112 & 768 & 0.01113 \\
\hline
\end{tabular}

Table 7: Errors in the solution as functions of the number of intervals. Problem (6.1). 
captures the solution and the discrete derivative with almost machine-accuracy (again, the observed breakdown of accuracy of the fourth-order scheme on finer grids is due to the loss of significant digits); see results in Table 8.

$$
\left(\beta u_{x}\right)_{x}=12 x^{2}, \quad \beta=\left\{\begin{array}{lll}
1, & \text { if } & 0 \leq x \leq 0.5 \\
2, & \text { if } & 0.5 \leq x \leq 1
\end{array}\right.
$$

subject to the boundary and interface conditions:

$$
\begin{aligned}
u(0) \equiv u_{1}(0)=0, & u(1) \equiv u_{2}(1)=\frac{17}{32} \\
u_{1}(0.5) & =u_{2}(0.5), \\
u_{1 x}(0.5) & =2 u_{2 x}(0.5)
\end{aligned}
$$

The exact solution is:

$$
u(x)=\left\{\begin{array}{lll}
u_{1}(x)=x^{4} & \text { if } & 0 \leq x \leq 0.5 \\
u_{2}(x)=\frac{1}{2}\left(x^{4}+\frac{1}{16}\right), & \text { if } & 0.5 \leq x \leq 1
\end{array}\right.
$$

\begin{tabular}{|c|c|c|}
\hline $\mathrm{N}$ & Solution Error (DPM 4) & Gradient(Derivative) Error (DPM 4) \\
\hline 24 & $3.809 \mathrm{e}-15$ & $1.466 \mathrm{e}-14$ \\
48 & $8.59 \mathrm{e}-15$ & $2.145 \mathrm{e}-13$ \\
96 & $3.819 \mathrm{e}-13$ & $1.266 \mathrm{e}-12$ \\
192 & $1.618 \mathrm{e}-12$ & $1.032 \mathrm{e}-11$ \\
384 & $2.404 \mathrm{e}-12$ & $5.165 \mathrm{e}-11$ \\
768 & $4.71 \mathrm{e}-11$ & $1.999 \mathrm{e}-10$ \\
\hline
\end{tabular}

Table 8: Errors in the solution and in the discrete derivative of the solution as functions of the number of intervals for DPM 4: we consider auxiliary domains [-0.667, 0.833] for $0 \leq x \leq 0.5$, and $[-0.167,1.33]$ for $0.5<x \leq 1$. Problem (6.6).

6.2. Second and fourth order difference potentials method for problem in heterogeneous media

In this section we consider the following test problem with variable coefficients:

$$
\left(k_{s} u_{s_{x}}\right)_{x}-\sigma_{s} u_{s}=f_{s}, \quad s=1,2
$$

with

$$
\begin{aligned}
& k_{1}(x)=3 e^{-10(x-0.5)^{4} x^{4}} \\
& k_{2}(x)=3 \\
& \sigma_{1}(x)=2 \\
& \sigma_{2}(x)=1
\end{aligned}
$$


subject to the boundary and interface conditions:

$$
\begin{aligned}
u(0) \equiv u_{1}(0)=0, & u(1) \equiv u_{2}(1)=1.0156 \\
u_{1}(0.5) & =u_{2}(0.5) \\
u_{1 x}(0.5) & =u_{2 x}(0.5)
\end{aligned}
$$

and

$$
u(x)=\left\{\begin{array}{lll}
u_{1}(x), & \text { if } & 0 \leq x \leq 0.5 \\
u_{2}(x), & \text { if } \quad 0.5 \leq x \leq 1
\end{array}\right.
$$

where the exact solution is given below

$$
\begin{aligned}
& u_{1}(x)=\sin (\pi x) \\
& u_{2}(x)=2(x-0.5)^{7}+1
\end{aligned}
$$

The $f_{s}$ are computed from the above equation. We have a variable coefficient $k_{1}(x)$ in subdomain $I_{1}$ and a constant coefficient $k_{2}$ in subdomain $I_{2}$. In Tables 9 - 14, we demonstrate overall second-order convergence for DPM 2 and fourth-order convergence for DPM 4 (6.10) - (6.13). However, the error does not converge monotonically, but rather oscillates for the variable coefficient problem. Again, we note that the breakdown of convergence of fourthorder scheme on finer grids is due to the loss of significant digits, as the absolute levels of error get very close to machine zero. In Tables 11 - 14, we select different grids for each subdomain. Results in Tables 11 and 13 show that we can take a coarser mesh in the subdomain with less oscillatory solution, while the error remains almost the same as in Tables 9 and 10. Similar results with the use of different grids in different subdomains are observed in 2D for the constant coefficient problem [27,4]. This illustrates the important flexibility of the method for the future development of the proposed ideas (multigrid/multiscale approach) for variable coefficient problems in $2 \mathrm{D}$ and $3 \mathrm{D}$.

\begin{tabular}{|c|c|c|c|c|c|}
\hline$N_{1}$ & $N_{2}$ & Error (DPM 2) & Conv. Rate & Error (DPM 4) & Conv. Rate \\
\hline 40 & 40 & 0.00018 & & $4.187 \mathrm{e}-06$ & \\
80 & 80 & $4.512 \mathrm{e}-05$ & 1.996 & $1.817 \mathrm{e}-07$ & 4.526 \\
160 & 160 & $1.133 \mathrm{e}-05$ & 1.994 & $2.417 \mathrm{e}-08$ & 2.910 \\
320 & 320 & $2.837 \mathrm{e}-06$ & 1.998 & $1.466 \mathrm{e}-09$ & 4.043 \\
640 & 640 & $7.091 \mathrm{e}-07$ & 2.000 & $9.334 \mathrm{e}-11$ & 3.973 \\
\hline
\end{tabular}

Table 9: Errors in the solution as functions of the number of intervals for DPM 2 and DPM 4: we consider auxiliary domains $[-0.167,0.583]$ with $N_{1}$ subintervals for $0 \leq x \leq 0.5$, and $[0.333,1.08]$ with $N_{2}$ subintervals for $0.5<x \leq 1$. Problem (6.10).

\section{Concluding remarks}

In this work, we used the one-dimensional elliptic type model with variable coefficients as the starting point, to develop and numerically test high-order methods based on Difference 


\begin{tabular}{|c|c|c|c|c|c|}
\hline$N_{1}$ & $N_{2}$ & Error (DPM 2) & Conv. Rate & Error (DPM 4) & Conv. Rate \\
\hline 48 & 48 & 0.0001253 & & $2.106 \mathrm{e}-06$ & \\
96 & 96 & $3.159 \mathrm{e}-05$ & 1.988 & $1.345 \mathrm{e}-07$ & 3.969 \\
192 & 192 & $7.862 \mathrm{e}-06$ & 2.007 & $2.617 \mathrm{e}-09$ & 5.684 \\
384 & 384 & $1.97 \mathrm{e}-06$ & 1.997 & $1.238 \mathrm{e}-09$ & 1.080 \\
768 & 768 & $4.918 \mathrm{e}-07$ & 2.002 & $1.079 \mathrm{e}-10$ & 3.520 \\
\hline
\end{tabular}

Table 10: Errors in the solution as functions of the number of intervals for DPM 2 and DPM 4: we consider auxiliary domains $[-0.167,0.583]$ with $N_{1}$ subintervals for $0 \leq x \leq 0.5$, and $[0.333,1.08]$ with $N_{2}$ subintervals for $0.5<x \leq 1$. Problem (6.10).

\begin{tabular}{|c|c|c|c|c|c|}
\hline$N_{1}$ & $N_{2}$ & Error (DPM 2) & Conv. Rate & Error (DPM 4) & Conv. Rate \\
\hline 80 & 40 & $7.242 \mathrm{e}-05$ & & $9.375 \mathrm{e}-08$ & \\
160 & 80 & $1.78 \mathrm{e}-05$ & 2.025 & $1.649 \mathrm{e}-08$ & 2.507 \\
320 & 160 & $4.467 \mathrm{e}-06$ & 1.995 & $9.082 \mathrm{e}-10$ & 4.182 \\
640 & 320 & $1.122 \mathrm{e}-06$ & 1.993 & $1.184 \mathrm{e}-10$ & 2.939 \\
\hline
\end{tabular}

Table 11: Errors in the solution as functions of the number of intervals for DPM 2 and DPM 4: we consider auxiliary domains $[-0.167,0.583]$ with $N_{1}$ subintervals for $0 \leq x \leq 0.5$, and $[0.333,1.08]$ with $N_{2}$ subintervals for $0.5<x \leq 1$. Problem (6.10).

\begin{tabular}{|c|c|c|c|c|c|}
\hline$N_{1}$ & $N_{2}$ & Error (DPM 2) & Conv. Rate & Error (DPM 4) & Conv. Rate \\
\hline 40 & 80 & 0.0001571 & & $4.275 \mathrm{e}-06$ & \\
80 & 160 & $3.959 \mathrm{e}-05$ & 1.989 & $1.894 \mathrm{e}-07$ & 4.496 \\
160 & 320 & $9.941 \mathrm{e}-06$ & 1.994 & $2.473 \mathrm{e}-08$ & 2.937 \\
320 & 640 & $2.487 \mathrm{e}-06$ & 1.990 & $1.655 \mathrm{e}-09$ & 3.901 \\
\hline
\end{tabular}

Table 12: Errors in the solution as functions of the number of intervals for DPM 2 and DPM 4: we consider auxiliary domains $[-0.167,0.583]$ with $N_{1}$ subintervals for $0 \leq x \leq 0.5$, and $[0.333,1.08]$ with $N_{2}$ subintervals for $0.5<x \leq 1$. Problem (6.10).

\begin{tabular}{|c|c|c|c|c|c|}
\hline$N_{1}$ & $N_{2}$ & Error (DPM 2) & Conv. Rate & Error (DPM 4) & Conv. Rate \\
\hline 96 & 48 & $5.085 \mathrm{e}-05$ & & $9.327 \mathrm{e}-08$ & \\
192 & 96 & $1.237 \mathrm{e}-05$ & 2.039 & $6.243 \mathrm{e}-09$ & 3.901 \\
384 & 192 & $3.134 \mathrm{e}-06$ & 1.981 & $1.111 \mathrm{e}-09$ & 2.490 \\
768 & 384 & $7.784 \mathrm{e}-07$ & 2.009 & $1.363 \mathrm{e}-10$ & 3.027 \\
\hline
\end{tabular}

Table 13: Errors in the solution as functions of the number of intervals for DPM 2 and DPM 4: we consider auxiliary domains $[-0.167,0.583]$ with $N_{1}$ subintervals for $0 \leq x \leq 0.5$, and $[0.333,1.08]$ with $N_{2}$ subintervals for $0.5<x \leq 1$. Problem (6.10). 


\begin{tabular}{|c|c|c|c|c|c|}
\hline$N_{1}$ & $N_{2}$ & Error (DPM 2) & Conv. Rate & Error (DPM 4) & Conv. Rate \\
\hline 48 & 96 & 0.000109 & & $2.147 \mathrm{e}-06$ & \\
96 & 192 & $2.776 \mathrm{e}-05$ & 1.973 & $1.393 \mathrm{e}-07$ & 3.946 \\
192 & 384 & $6.879 \mathrm{e}-06$ & 2.013 & $2.491 \mathrm{e}-09$ & 5.805 \\
384 & 768 & $1.727 \mathrm{e}-06$ & 1.994 & $1.476 \mathrm{e}-09$ & 0.755 \\
\hline
\end{tabular}

Table 14: Errors in the solution as functions of the number of intervals for DPM 2 and DPM 4: we consider auxiliary domains $[-0.167,0.583]$ with $N_{1}$ subintervals for $0 \leq x \leq 0.5$, and $[0.333,1.08]$ with $N_{2}$ subintervals for $0.5<x \leq 1$. Problem (6.10).

Potentials approach for the variable coefficient elliptic problems in heterogeneous media. We also illustrated the unified framework (principles) for the construction of Difference Potentials Methods with high-order accuracy for the single domain, and for the interface/composite domain problems with non-matching interface conditions. While the methods and analysis are simple for these one-dimensional problems, they allow us to illustrate and test several ideas and capabilities of high-order methods based on Difference Potentials approach. The numerical schemes, as well as meshes can be chosen totally independently for each subdomain/ domain; in higher-dimensions the boundaries of the subdomains and interfaces do not need to conform/align with the grids. We expect that the high-order schemes can be constructed for problems with general boundary conditions, and the main complexity of the developed algorithm reduces to the several solutions of simple auxiliary problems on structured Cartesian grids. Also, the preliminary tests that we conducted here in the one-dimensional settings (as well as preliminary 2D numerical tests in $[23,27,4,3]$ ) indicate the capability of Difference Potentials approach to resolve discontinuities very accurately at the interface. Therefore, we expect that the proposed approach will be well-suited for the general heterogeneous models and interface problems.

For future research, we plan to extend and further develop the proposed approach (as well as methods that we developed in [23, 27, 4, 3]) to high-order methods for variable coefficient problems in arbitrary domains in $2 D$ and $3 D$, including the time-dependent problems. The high-order Difference Potentials methods for time-dependent problems will be developed by considering the time-discrete version of the continuous problems (employing Backward Euler, Crank-Nicolson or high-order IMEX, etc. time discretizations; see some examples of $2 D$ second order in space and first order in time DPM schemes in [3, 4]). We also plan to develop iterative solvers, for example a multigrid iterative solver for the efficient solution of the auxiliary problems in each subdomain/domain, see some references on multigrid [6, 7], [1], [8], [29] and other references. We expect that the developed approach will be well-suited for multi-physics/multi-scale problems with general boundary conditions, as well as for the development of parallel algorithms.

\section{Acknowledgment:}

The authors are grateful to the anonymous referees for the valuable suggestions and questions that helped to improve the manuscript. 
The research of Yekaterina Epshteyn is supported in part by the National Science Foundation Grant \# DMS-1112984 and the research of Spencer Phippen is supported in part by

the National Science Foundation Grant \# DMS-1112984 and by the REU research at the Mathematics Department at the University of Utah.

\section{Appendix}

For the reader's convenience, similar to the second-order method in the case of a constant coefficient model problem (4.1) (assume, $k(x) \equiv 1$ ), let us show a direct connection of the difference potential $\mathbf{P}_{N^{+} \gamma} u_{\gamma}$ to the Cauchy-type integral. Again, we will assume $\sigma(x)=0$.

The homogeneous difference equation $L_{h}\left[u_{j}\right]=0, \quad j=l+2, \ldots, L+1$ for the fourth order scheme is

$$
\frac{-u_{j-2}+16 u_{j-1}-30 u_{j}+16 u_{j+1}-u_{j+2}}{12 h^{2}}=0, \quad j=l+2, \ldots, L+1
$$

Consider the difference equation of the form

$$
-z_{j-2}+16 z_{j-1}-30 z_{j}+16 z_{j+1}-z_{j+2}=0
$$

Denote, $z:=\left(z_{l}, z_{l+1}, . ., z_{L+2}, z_{L+3}\right)$ to be a solution of the difference equation (8.2). Next, define the generating polynomial

$$
Z(g)=\sum_{j=l}^{L+3} z_{j} g^{j}
$$

where the coefficients of the polynomial $z_{j}$ are the values of the solution $z$ at the grid points. Multiply (8.2) by $g^{j}$, and sum from $l+2, \ldots, L+1$ to obtain:

$$
\sum_{j=l+2}^{L+1} z_{j-2} g^{j}-16 \sum_{j=l+2}^{L+1} z_{j-1} g^{j}+30 \sum_{j=l+2}^{L+1} z_{j} g^{j}-16 \sum_{j=l+2}^{L+1} z_{j+1} g^{j}+\sum_{j=l+2}^{L+1} z_{j+2} g^{j}=0
$$

As for the second-order method in Section 4.1, we can rewrite each term. For example, we can rewrite the first term as:

$$
g^{2} \sum_{j=l+2}^{L+1} z_{j-2} g^{j-2}=g^{2} Z(g)-z_{L} g^{L+2}-z_{L+1} g^{L+3}-z_{L+2} g^{L+4}-z_{L+3} g^{L+5} .
$$

Similarly, the other terms in (8.3) can be rewritten as well. Again, let us recall Cauchy's residue theorem, and represent

$$
z_{j}=\frac{1}{2 \pi i} \oint_{|g|=2} \frac{Z(g)}{g^{j+1}} d g
$$


Thus, we obtain

$$
\begin{aligned}
z_{j} & =\frac{1}{2 \pi i} \oint_{|g|=2} \frac{\left(1-16 g+30 g^{2}-16 g^{3}\right) g^{l} z_{l}+\left(1-16 g+30 g^{2}\right) g^{l+1} z_{l+1}+(1-16 g) g^{l+2} z_{l+2}}{(g-1)^{2}\left(g^{2}-14 g+1\right) g^{j+1}} d g \\
& +\frac{1}{2 \pi i} \oint_{|g|=2} \frac{g^{l+3} z_{l+3}+g^{L+4} z_{L}+(-16+g) g^{L+4} z_{L+1}+\left(30-16 g+g^{2}\right) g^{L+4} z_{L+2}}{(g-1)^{2}\left(g^{2}-14 g+1\right) g^{j+1}} d g \\
& +\frac{1}{2 \pi i} \oint_{|g|=2} \frac{\left(-16+30 g-16 g^{2}+g^{3}\right) g^{L+4} z_{L+3}}{(g-1)^{2}\left(g^{2}-14 g+1\right) g^{j+1}} d g, \quad j=l, \ldots, L+3
\end{aligned}
$$

The Cauchy - type integral (8.4) plays the role of the discrete potential for the difference equation (8.2) (each $z_{j}, j=l, \ldots, L+3$ is determined by values $z_{\gamma}$ ), similar to the difference potential $\mathbf{P}_{N^{+} \gamma} u_{\gamma}$ for (8.1).

\section{References}

[1] A. Brandt, Multi-level adaptive solutions to boundary-value problems, Math. Comp. 31 (138) (1977) 333-390.

[2] D. Britt, S. Tsynkov, E. Turkel, A high-order numerical method for the helmholtz equation with nonstandard boundary conditions, SIAM Journal on Scientific Computing 35 (5) (2013) A2255-A2292.

URL http://epubs.siam.org/doi/abs/10.1137/120902689

[3] Y. Epshteyn, Upwind-difference potentials method for Patlak-Keller-Segel chemotaxis model, Journal of Scientific Computing 53 (3) (2012) 689 - 713, dOI: 10.1007/s10915012-9599-2.

[4] Y. Epshteyn, Algorithms Composition Approach based on Difference Potentials Method for Parabolic ProblemsCommunications in Mathematical Sciences, to appear.

[5] R. P. Fedkiw, T. Aslam, B. Merriman, S. Osher, A non-oscillatory Eulerian approach to interfaces in multimaterial flows (the ghost fluid method), J. Comput. Phys. 152 (2) (1999) 457-492.

URL http://dx.doi.org/10.1006/jcph.1999.6236

[6] R. P. Fedorenko, A relaxation method of solution of elliptic difference equations, Ž. Vyčisl. Mat. i Mat. Fiz. 1 (1961) 922-927.

[7] R. P. Fedorenko, On the speed of convergence of an iteration process, Ž. Vyčisl. Mat. i Mat. Fiz. 4 (1964) 559-564.

[8] M. Holst, F. Saied, Parallel performance of some multigrid solvers for three-dimensional parabolic equations, Tech. rep. (1991).

[9] R. J. LeVeque, Z. Li, Immersed interface methods for Stokes flow with elastic boundaries or surface tension, SIAM J. Sci. Comput. 18 (3) (1997) 709-735.

URL http://dx.doi.org/10.1137/S1064827595282532 
[10] R. J. LeVeque, Z. L. Li, The immersed interface method for elliptic equations with discontinuous coefficients and singular sources, SIAM J. Numer. Anal. 31 (4) (1994) 1019-1044.

URL http://dx.doi.org/10.1137/0731054

[11] Z. Li, K. Ito, The immersed interface method, vol. 33 of Frontiers in Applied Mathematics, Society for Industrial and Applied Mathematics (SIAM), Philadelphia, PA, 2006, numerical solutions of PDEs involving interfaces and irregular domains.

URL http://dx.doi.org/10.1137/1.9780898717464

[12] X.-D. Liu, R. P. Fedkiw, M. Kang, A boundary condition capturing method for Poisson's equation on irregular domains, J. Comput. Phys. 160 (1) (2000) 151-178.

URL http://dx.doi.org/10.1006/jcph.2000.6444

[13] X.-D. Liu, T. C. Sideris, Convergence of the ghost fluid method for elliptic equations with interfaces, Math. Comp. 72 (244) (2003) 1731-1746 (electronic). URL http://dx.doi.org/10.1090/S0025-5718-03-01525-4

[14] J. Loncaric, V. S. Ryaben'kii, S. V. Tsynkov, Active shielding and control of noise, SIAM J. Appl. Math. 62 (2) (2001) 563-596 (electronic).

[15] A. Mayo, The fast solution of Poisson's and the biharmonic equations on irregular regions, SIAM J. Numer. Anal. 21 (2) (1984) 285-299.

URL http://dx.doi.org/10.1137/0721021

[16] M. Medvinsky, S. Tsynkov, E. Turkel, The method of difference potentials for the helmholtz equation using compact high order schemes, Journal of Scientific Computing (May 2012) 1-44.

URL http: //www. scopus. com/inward/record.url?eid=2-s2. 0-84860903077\&partner ID $=40 \& m d 5=915737 f 56$ f ce 5 f $42 \mathrm{cbc711491887a605}$

[17] C. S. Peskin, Numerical analysis of blood flow in the heart, J. Computational Phys. 25 (3) (1977) 220-252.

[18] C. S. Peskin, The immersed boundary method, Acta Numer. 11 (2002) 479-517. URL http://dx.doi.org/10.1017/S0962492902000077

[19] A. A. Reznik, Approximation of surface potentials of elliptic operators by difference potentials, Dokl. Akad. Nauk SSSR 263 (6) (1982) 1318-1321.

[20] A. A. Reznik, Approximation of surface potentials of elliptic operators by difference potentials and solution of boundary value problems, Ph.D, Moscow, MPTI.

[21] V. S. Rjaben'kiū, Computation of heat conductivity on a system of rods, Ž. Vyčisl. Mat. i Mat. Fiz. 10 (1970) 236-239.

[22] V. Ryaben'kii, Method of difference potentials and its applications, Springer-Verlag. 
[23] V. Ryaben'kii, V. I. Turchaninov, Y. Y. Epshteyn, The numerical example of algorithms composition for solution of the boundary-value problems on compound domain based on difference potential method, Moscow, Keldysh Institute for Applied Mathematics, Russia Academy of Sciences (3).

[24] V. S. Ryaben'kiü, Difference potentials analogous to Cauchy integrals, Uspekhi Mat. Nauk 67 (3(405)) (2012) 147-172.

[25] V. S. Ryaben'kii, S. V. Tsynkov, Artificial boundary conditions for the numerical solution of external viscous flow problems, SIAM J. Numer. Anal. 32 (5) (1995) 1355-1389.

[26] V. S. Ryaben'kii, S. V. Tsynkov, S. V. Utyuzhnikov, Active control of sound with variable degree of cancellation, Appl. Math. Lett. 22 (12) (2009) 1846-1851.

URL http://dx.doi.org/10.1016/j.aml.2009.07.010

[27] V. S. Ryaben'kiı̆, V. I. Turchaninov, E. Y. Èpshtĕn, An algorithm composition scheme for problems in composite domains based on the method of difference potentials, Zh. Vychisl. Mat. Mat. Fiz. 46 (10) (2006) 1853-1870.

URL http://dx.doi.org/10.1134/S0965542506100137

[28] S. Tsynkov, Numerical solution of problems on unbounded domains. A review, Appl. Numer. Math. 27 (4) (1998) 465-532, absorbing boundary conditions.

[29] J. Van Lent, S. Vandewalle, Multigrid methods for implicit Runge-Kutta and boundary value method discretizations of parabolic PDEs, SIAM J. Sci. Comput. 27 (1) (2005) $67-92$.

URL http://dx.doi.org/10.1137/030601144

[30] S. Yu, Y. Zhou, G. W. Wei, Matched interface and boundary (MIB) method for elliptic problems with sharp-edged interfaces, J. Comput. Phys. 224 (2) (2007) 729-756.

URL http://dx.doi.org/10.1016/j.jcp.2006.10.030

[31] Y. C. Zhou, J. Liu, D. L. Harry, A matched interface and boundary method for solving multi-flow Navier-Stokes equations with applications to geodynamics, J. Comput. Phys. 231 (1) (2012) 223-242.

URL http://dx.doi.org/10.1016/j.jcp.2011.09.010

[32] Y. C. Zhou, S. Zhao, M. Feig, G. W. Wei, High order matched interface and boundary method for elliptic equations with discontinuous coefficients and singular sources, J. Comput. Phys. 213 (1) (2006) 1-30.

URL http://dx.doi.org/10.1016/j.jcp.2005.07.022 